\title{
Theory of frozars and its observable effects. 1. Structure of stars frozen during general relativistic collapse
}

\begin{abstract}
Zahid Zakir ${ }^{1}$
Abstract

As a star collapses, positions of its particles, as for any extended object, must be set on the hypersurfaces of simultaneity $t=$ const., marked by world time moments $t$, i.e. ordinary astronomical time around a star. Then the surface of a dust star freezes over its gravitational radius and such asymptotic behaviour of the worldlines of star's particles on the surface is invariant. The star's center freezes before other layers, after which the entire structure of the star quickly freezes. This means that a specifically general relativistic phenomenon - gravitational time dilation - is the physical mechanism that stops the collapse in terms of t. Such freezing is shown for exactly solvable models. A thin shell freezes outside its gravitational radius, its interior remains flat, and the test particles inside also freeze. A homogeneous dust star, as shows the Oppenheimer-Snyder solution in terms t, becomes a frozen star or frozar. The inner layers remain locally homogeneous and freeze near their asymptotes. Before the freezing, sufficiently massive stars have a density below a neutron star and, therefore, if their nuclei have not exploded before, the collapse of such stars occur like a dust star with the frozar formation. The rotation of stars freezes even before the surface reaches the ergosphere boundary, so the rotated frozar has not a horizon and an ergosphere. Accretion to frozar leads to freezing of the falling matter above the surface with formation of an inhomogeneous landscape of flattened mascons. Frozars do not merge, but only stick together near the gravitational radius of the multifrozar system, by forming, together with ordinary matter, a frozar cluster. Supermassive frozars, or superfrozars, are formed mainly as such heterogeneous clusters. Frozars and their clusters are not "bald", but may have a "hairstyle" and an asymmetric structure. The inhomogeneities of their field can be detected by gravimetry, inhomogeneities of shadows, redshifts and orbits of matter. Observational consequences and prospects of the frozar theory are discussed.
\end{abstract}

Keywords: gravitational collapse, frozen stars, frozars, black holes, horizon, singularity, ergosphere, accretion, gravitational waves, relativistic stars

\section{Content}

Introduction

1. The collapse of a thin dust shell with the empty frozar formation ...........................................4

1.1. Trajectories of shell particles in an external static metric ............................................ 4

1.2. $\quad$ The relationship between the shell proper time and world time .................................. 6

1.3. Freezing of the inner region and lack of horizon and singularity ................................. 8

1.4. Frozar formation is independent on the choice of coordinates ..................................... 8

2. Exact solutions for a spherical homogeneous dust star .......................................................11

2.1. Friedmann-Tolman solution in comoving coordinates .............................................. 11

2.2. Transformation of the solutions from comoving to static coordinates.......................... 12

2.3. Oppenheimer-Snyder solution for a uniform dust star .............................................. 13

2.4. Worldlines of particles in the layers and their asymptotes ......................................... 15

3. Freezing at the collapse of charged and rotating stars ......................................................17

3.1. Collapse of a charged spherical star .................................................................... 17

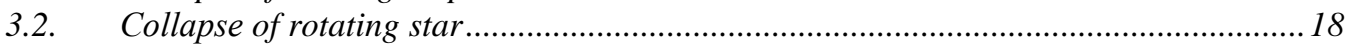

4. Heterogeneity of structure and sticking together of frozars ..............................................19

${ }^{1}$ Center for Theoretical Physics and Astrophysics, Tashkent Uzbekistan, zzakir@ qgph.org, ORCID 
4.1. Freezing of more than half of the frozar mass close to surface ...................................19

4.2. Formation of the accretionary landscape on the frozars ........................................20

4.3. Sticking together of frozars without merging ..............................................................21

4.4. Supermassive frozars (superfrozars) as heterogeneous clusters..................................22

5. Observable effects of theory of frozars ............................................................................23

5.1. Observable properties of frozars and explanation of known effects ...........................22

5.2. Effects of surface heterogeneity and internal structure ...............................................24

5.3. Consequences of the absence of an ergosphere in rotated frozars ...............................24

5.4. The effects of sticking of frozars without merging ....................................................25

5.5. Effects of a delay of falling the charged surface layer.............................................25

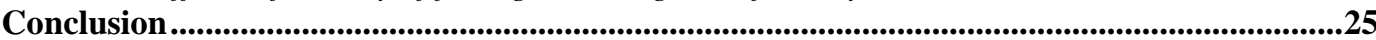

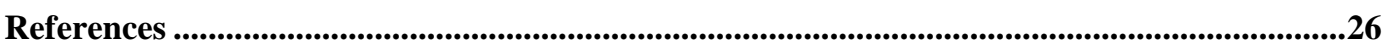

\section{Introduction}

The collapse of a dust star in general relativity can be described in terms of two types of time, where each of them expresses only one of the two main properties of physical time. A description of the collapse in terms of proper time $\tau$, expressing the local rate of physical processes, has long been considered as a standard one. But this time is not suitable for describing the entire star as a set of simultaneously coexisting particles. The latter is realized only in the description in world time $t$ expressing the global simultaneity of events both inside and outside the star.

At such description, the surface of the collapsing star hangs over its gravitational radius $r_{g}=2 m G$ at any moment $t<\infty$, where $G$ is gravitational constant. Freezing of the surface means that $\tau$ almost stops going in terms of $t$ and at $t \rightarrow \infty$ tends to a finite value $\tau(t) \rightarrow \tau_{g}$ (see (18)).

Due to the difference in the upper limits of the two forms of physical time, there have been attempts to continue the $\tau$-description beyond $\tau_{g}$ and to describe mythical "events" in the non-physical region $t>\infty$. The star's surface was attributed by a mythical ability to cross $r_{g}$ and reach the center in a finite interval of $\tau$, forming a singularity. This area of mathematical physics became known as the black hole theory and was widely promoted. However, it is not related to real stars in the physical world existing at $t<\infty$ only.

At describing the star at $t<\infty$, when the proper times of its particles also are in the physical region $\tau<\tau_{g}$, the question of choosing one of two types of time has a more practical sense and is not as fateful as in the above mentioned non-physical experiment. Events on the worldlines of star's particles are parametrized both $t$ and $\tau$, thus their moments, describing the same event, are uniquely related across the identity $r(R, \tau)=r(R, t)$, following from identity of the solutions of two equations of motion $(R$ is the initial value of $r$ at $\tau=0)$. The relationship between these two times is discussed below in Section 1.2.

The structure and evolution of a star in this physical picture is determined from an analysis of the exact solutions of the field equations and the equations of motion in a simple and realistic model under physically correct initial and boundary conditions. In this case, it is a model of a locally homogeneous spherical dust star, particles of which freely fall radially. A spherical star is most easily described in the rest frame of its center, where the external metric on the hypersurface $t=$ const. of this global static frame is the 
Schwarzschild metric. Therefore, for matching with the external metric, the internal metric must also be presented in the same static frame.

For the parabolic velocity of the dust star particles $(k=0)$, this problem was solved by Oppenheimer and Snyder (OS) [1] by transforming the Friedmann solution (in Tolman's form [2]) into the Schwarzschild coordinates $r, t$. For the diagonal metric, when it is possible to uniquely determine the simultaneity inside the star, they obtained the simplest solution for the metric and equations of motion $t=M[y(R, r)]$. Here an auxiliary function $y(R, r)$ was introduced, which was taken in the form:

$$
y(R, r)=\frac{1}{2}\left(\frac{R^{2}}{R_{b}^{2}}-1\right)+\frac{R_{b}}{R r_{g}} r,
$$

where $R_{b}, R$ - are the initial radial coordinates of the surface and layer. The function $M$ was determined from the matching condition on the surface. A detailed derivation of the form of the function $y(R, r)$ was given in [3-4]. The OS solution was the first one which allowed to describe the instantaneous structure of the star as a set of simultaneous events.

A solution for the elliptic velocity of the dust star particles $(k=1)$ was found by $\mathrm{O}$. Klein [5] and S. Weinberg [6] by using two other methods of diagonalization of the transformed metric. Then, in the second of the papers [3], the solution for all three special speeds $(k=0, \pm 1)$, preserving uniformity during evolution, was obtained by the OS method. A complete solution of the dust star collapse problem for three speeds by these three methods, OS, Klein and Weinberg, is presented in the second paper [7].

In the papers [3-4,8-9], a new treatment of stellar collapse was developed, based on the specifics of two types of physical time in GR. It is taken into account that the $\tau$ description has a physical meaning for the entire star only as a part of the $t$-description on the hypersurfaces of simultaneity $t=$ const., where the star is a set of simultaneously coexisting particles. The collapsed object in this treatment become a completely frozen star (frozar) ${ }^{1}$, as it is demonstrated in the case of OS solution.

The aims of this first paper are, firstly, a detailed derivation of the OS solution and analysis of its consequences with visualization of the worldlines in the layers and the star's structure, secondly, the study of the properties of frozars, including charged and rotating ones, and thirdly, the discussion of the observational effects of frozars and their clusters. A more details of the frozar theory will be presented in the book [11].

In Section 1 the collapse of a thin dust shell is described. In Section 2 the OS solution is derived and the frozar formation is demonstrated. In Section 3 the charged and rotated frozars are discussed. In Section 4 the sticking together of frozars without merging and the heterogeneity of massive frozars are considered. In Section 5 the observational consequences of the theory of frozars are discussed.

\footnotetext{
${ }^{1}$ In my earlier publications, the frozar formation was shown firstly for a thin dust shell [8], and then for a star in some approximations [9]. Discussions with a more popular presentation are presented in [10].
} 


\section{The collapse of a thin dust shell with the empty frozar formation}

\subsection{Trajectories of shell particles in an external static metric}

In Newtonian gravity, the radial distribution in a spherical dust star does not affect to its external gravitational field, including the field on the surface. Therefore, if, without changing the total mass, a star inside is made empty, compressing all its matter on the surface in the form of a thin dust shell, then the field outside the star and on the surface will remain the same. In this case, the potential of the field inside the shell star will be constant and equal to the value of the potential on the surface.

In general relativity there is Birkhoff's theorem according to which the situation here is the same as in the Newtonian case. The field on the shell and outside is the Schwarzschild field, the space inside the shell is flat, the time component of the internal metric is the same at any point and equal to the surface value. Thus, the thin dust shell model is the simplest exactly solvable model, showing the basic properties of the general relativistic collapse of stars. Below we will study the relativistic collapse in the case of this simplest model.

The line element on the spherical shell and outside it in the static coordinates $(t, r, \theta, \varphi)$ is given by the Schwarzschild metric:

$$
d s^{2}=\alpha_{r} d t^{2}-\alpha_{r}^{-1} d r^{2}-r^{2} d \Omega^{2}, \quad r \geq r_{b}(t),
$$

where $\alpha_{r} \equiv 1-r_{g} / r, d \Omega^{2}=d \theta^{2}+\sin ^{2} \theta d \varphi^{2}, r_{g}=2 m G$ and $r_{b}(t)$ is the radius of the circumference of the shell (in the notations of OS [1] and [12]). Inside the shell, the line element has the form:

$$
d s^{2}=\left[1-r_{g} / r_{b}(t)\right] d t^{2}-d r^{2}-r^{2} d \Omega^{2}, \quad r<r_{b}(t) .
$$

Each of the shell particles falls in the field of the entire shell and moves like a test particle in the static metric (1). Therefore, to describe the evolution of the shell, it is enough to consider the trajectory of the test particle on the shell.

At radial falling of a particle rested at $r \rightarrow \infty$, the local velocity $v_{r}$ is parabolic and the energy conservation condition gives for it:

$$
\frac{\alpha_{r}^{1 / 2}}{\left(1-v_{r}^{2}\right)^{1 / 2}}=1, \quad v_{r}=\frac{1}{\alpha_{r}} \frac{d r}{d t}=-\frac{r_{g}^{1 / 2}}{r^{1 / 2}} .
$$

Let $r=R$ correspond to zero proper time moment $\tau(R, R)=0$. Then the proper time interval of the particle (1) and the following solution for $\tau(R, r)$ take the form:

$$
\begin{gathered}
d \tau^{2}=\left(1-r / r_{g}\right)\left(1-v_{r}^{2}\right) d t^{2}=\frac{r}{r_{g}} d r^{2} . \\
\tau(R, r)=\frac{2}{3 r_{g}^{1 / 2}}\left(R^{3 / 2}-r^{3 / 2}\right), \quad r(\tau, R)=\left(R^{3 / 2}-\frac{3}{2} r_{g}^{1 / 2} \tau\right)^{2 / 3} .
\end{gathered}
$$

The expression for the world time moment $t$ also follows from (4):

$$
t(R, r)-t_{0}(R)=\frac{1}{r_{g}^{1 / 2}} \int_{r(t)}^{R} \frac{d r r^{3 / 2}}{r-r_{g}},
$$


where $t_{0}(R)$ is the value of $t$ corresponding to the moment $\tau=0$ at $r=R$. The values of the integral (7) give the particle's worldline $r(R, t)$ in the implicit form $t(R, r)$ :

$$
t-t_{0}(R)=\frac{2}{3 r_{g}^{1 / 2}}\left(R^{3 / 2}-r^{3 / 2}\right)+2 r_{g}^{1 / 2}\left(R^{1 / 2}-r^{1 / 2}\right)+r_{g} \ln \left(\frac{r^{1 / 2}+r_{g}^{1 / 2}}{r^{1 / 2}-r_{g}^{1 / 2}} \frac{R^{1 / 2}-r_{g}^{1 / 2}}{R^{1 / 2}+r_{g}^{1 / 2}}\right) .
$$

Zero initial values $t=0$ for the synchronized world time clocks are given on the hypersurface of simultaneity $r=\bar{R}$. In this case, in (7), the upper limit of the integral $R$ is replaced by $\bar{R}$ and $t_{0}(\bar{R})=0$, i.e. there are no initial shifts in the times of the layers. The worldline $t(\bar{R}, r)$, as a result, is given by the expression:

$$
t=\frac{2}{3 r_{g}^{1 / 2}}\left(\bar{R}^{3 / 2}-r^{3 / 2}\right)+2 r_{g}^{1 / 2}\left(\bar{R}^{1 / 2}-r^{1 / 2}\right)+r_{g} \ln \left(\frac{r^{1 / 2}+r_{g}^{1 / 2}}{r^{1 / 2}-r_{g}^{1 / 2}} \frac{\bar{R}^{1 / 2}-r_{g}^{1 / 2}}{\bar{R}^{1 / 2}+r_{g}^{1 / 2}}\right) .
$$

Subtracting (8) from (9) we find the expression for the shift $t_{0}(R)$ (here $R=R(\bar{R})$ ):

$$
t_{0}(R)=\frac{2}{3 r_{g}^{1 / 2}}\left(\bar{R}^{3 / 2}-R^{3 / 2}\right)+2 r_{g}^{1 / 2}\left(\bar{R}^{1 / 2}-R^{1 / 2}\right)+r_{g} \ln \left(\frac{\bar{R}^{1 / 2}-r_{g}^{1 / 2}}{\bar{R}^{1 / 2}+r_{g}^{1 / 2}} \frac{R^{1 / 2}+r_{g}^{1 / 2}}{R^{1 / 2}-r_{g}^{1 / 2}}\right)
$$

From (6), using the condition $\tau^{\prime}=0$ on the hypersurfaces of the "same age" $\tau=$ const. , we find $\dot{r} \equiv \partial r / \partial \tau, r^{\prime} \equiv \partial r / \partial R$, and from (8) we calculate $\dot{t} \equiv \partial t / \partial \tau$ :

$$
\dot{r}=-\frac{r_{g}^{1 / 2}}{r^{1 / 2}}, \quad r^{\prime}=\frac{R^{1 / 2}}{r^{1 / 2}}, \quad \dot{t}=\alpha_{r}^{-1} .
$$

For finding $t^{\prime} \equiv \partial t / \partial R$ it is required an explicit value of $t_{0}(R)$. The initial radial coordinates of $R$ are fixed at $\tau=0$ in the comoving frame, where the metric is diagonal, and the metric diagonality condition gives (see below (46)) [1]:

$$
t^{\prime}=\dot{t} \dot{r} r^{\prime}=-\frac{r_{g}^{1 / 2}}{r} \cdot \frac{R^{1 / 2}}{1-r_{g} / r}=-\frac{\left(r_{g} R\right)^{1 / 2}}{r-r_{g}} .
$$

The integration in (12) gives a solution for the worldline $r(R, t)$ in the OS form [1]:

$$
t=\frac{2}{3 r_{g}^{1 / 2}}\left(R^{3 / 2}-r^{3 / 2}\right)-2 r_{g}^{1 / 2} r^{1 / 2}+r_{g} \ln \frac{r^{1 / 2}+r_{g}^{1 / 2}}{r^{1 / 2}-r_{g}^{1 / 2}} .
$$

Comparing (13) with (8) we find a solution for $t_{0}(R)$ :

$$
t_{0}(R)=-2 r_{g}^{1 / 2} R^{1 / 2}-r_{g} \ln \frac{R^{1 / 2}-r_{g}^{1 / 2}}{R^{1 / 2}+r_{g}^{1 / 2}} .
$$

Equating the right sides of (14) and (10) we find the relation between $R$ and $\bar{R}$ :

$$
R^{3 / 2}=\bar{R}^{3 / 2}\left[1+\frac{3 r_{g}}{\bar{R}}-\frac{3 r_{g}^{3 / 2}}{2 \bar{R}^{3 / 2}} \ln \left(\frac{\bar{R}^{1 / 2}+r_{g}^{1 / 2}}{\bar{R}^{1 / 2}-r_{g}^{1 / 2}}\right)\right]
$$


As we see, at large $\bar{R}$ the difference with $R$ is small, the values $t_{0}(R)$ are also small, and then, in the $t$-description, one can use the simpler formulas with $R$.

\subsection{The relationship between the shell proper time and world time}

The evolution of the shell in world time, like any star, is a transition from one hypersurface $t=t_{1}$ to another $t=t_{2}$. The worldlines of dust particles between the hypersurfaces of the same world time intervals $\Delta t=t_{2}-t_{1}$, correspond different intervals of proper time $\Delta \tau(r, t)$, as smaller, as closer the surface to $r_{g}$, which expresses the time dilation, the slowdown of $\tau$ with respect to uniformly going $t$.

Diagrams of worldlines $r(R, t)$ in accordance with (13) and $r(R, \tau)$ in accordance with (6) at $t<\infty$ are shown in Fig. 1. It is important here that at $r>r_{g}$ there is a one-to-one correspondence between both curves representing the same events on the same worldline of the same particle

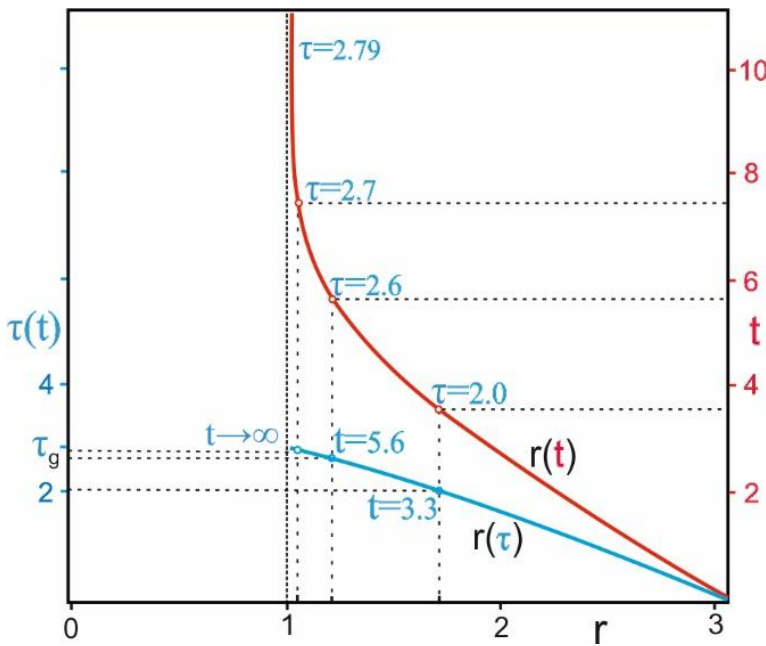

Fig. 1. The worldline of a falling test particle in terms of world time $t$ and proper time $\tau$. $r(R, t)=r(R, \tau)$.

The dependence $\tau(R, t)$ follows from (6) and (13) in the implicit form $t(R, \tau)$ :

$$
t=\tau-2\left(r_{g} R\right)^{1 / 2}\left(1-\frac{3 r_{g}^{1 / 2} \tau}{2 R^{3 / 2}}\right)^{1 / 3}+r_{g} \ln \frac{\left(1-3 r_{g}^{1 / 2} \tau / 2 R^{3 / 2}\right)^{1 / 3}+\frac{r_{g}^{1 / 2}}{R^{1 / 2}}}{\left(1-3 r_{g}^{1 / 2} \tau / 2 R^{3 / 2}\right)^{1 / 3}-\frac{r_{g}^{1 / 2}}{R^{1 / 2}}} .
$$

It is clearly seen here that at $r>r_{g}$ (and fixed $\varphi, \theta$ ) there is a one-to-one correspondence (16) between the moments of $t$ and $\tau(R, t)$.

At $t \gg r_{g}$ the particle asymptotically approaches $r_{g}$, i.e. $r \simeq r_{g}+h, h \ll r_{g}$ and both expressions for its worldline (6) and (13) are extremely simplified:

$$
\begin{gathered}
t \simeq b(R)+r_{g} \ln \frac{r_{g}}{h}, \quad h \simeq \bar{b} \exp \left(-t / r_{g}\right), \\
\tau(R, h) \simeq \tau_{g}(R)-h, \quad \tau_{g}=\frac{2}{3 r_{g}^{1 / 2}}\left(R^{3 / 2}-r_{g}^{3 / 2}\right),
\end{gathered}
$$

where $b \equiv 2\left(R^{3 / 2}-4 r_{g}^{3 / 2}\right) / 3 r_{g}^{1 / 2}, \bar{b} \equiv r_{g} \exp \left(b / r_{g}\right)$. Thus, at $r \simeq r_{g}+h$, both curves $t(R, r)$ and $\tau(R, r)$ in Fig. 1, describing the particle's worldline $r(R, t)=r(R, \tau)$, are 
asymptotic. Expressing $h$ from (18), and substituting into (17), we obtain the relation of two times $t(R, \tau)$ and $\tau(R, t)$ in asymptotics:

$$
t \simeq b+r_{g} \ln \frac{4 r_{g}}{\tau-\tau_{g}}, \quad \tau(t) \simeq \tau_{g}-\bar{b} \exp \left(-t / r_{g}\right) .
$$

Let's consider the limit at $r \rightarrow r_{g}$, i.e. $h \rightarrow 0$. For $t$ from (17), it follows $t \rightarrow \infty$, i.e. the falling particle freezes asymptotically near $r_{g}$, never reaching it. Correspondingly, the proper time of the particle also freezes asymptotically $\tau(t) \rightarrow \tau_{g}$, approaching, but never reaching (at $t<\infty$.) the freezing moment $\tau_{g}$ from (18).

Thus, the events on the worldline $t(R, r)$ in an infinite interval of world time $t \in\left[t_{0}, \infty\right)$ one-to-one correspond to events on the worldline $\tau(R, r)$ in a finite interval of proper time $\tau \in\left[0, \tau_{g}\right)$. In the Newtonian theory both intervals of change, for $t$ and $\tau$, were infinite, $t \in\left[t_{0}, \infty\right)$ and $\tau \in[0, \infty)$, what made them identical. But the existence of time dilation (gravitational and kinematical) means that one of the two intervals, for proper time, becomes finite and is replaced by $\tau \in\left[0, \tau_{g}\right)$. Notice, that the points of the finite proper time interval $\left[0, \tau_{g}\right)$ continue to be in the one to one correspondence with the points of the infinite world time interval $\left[t_{0}, \infty\right)$, which is not surprising for continuous sets.

Thus, the $t$-description covers all the events on the worldline of the particle $t(R, r)$ for the entire time $t<\infty$ as the star exists in the real universe and therefore it is complete. Equally complete is the $\tau$-description in the interval $\left[0, \tau_{g}\right)$, being another parameterization $\tau(R, r)$ of the events on the same worldline $t(R, r)$.

In the non-physical treatments of collapse it was declared that the worldline of a particle in terms of $\tau$ can reach $r_{g}$ and cross it. In general relativity this mathematical extrapolation turns out to be an artifact of Newtonian theory, not related to the solutions of the general relativistic equations of motion, where $\tau$ changes only in the interval $\left[0, \tau_{g}\right.$ ) during the entire life of the star in the real world with $t<\infty$. In general relativity, the region of events $\tau \geq \tau_{g}$, corresponding to $t \geq \infty$, turns out to be nonphysical and not related to the worldline of a real particle.

An artifact of the Newtonian theory is also the intuitive statement of mathematical treatments that if the worldline $r(R, \tau)$ in Fig. 1 does not cross $r_{g}$, then this apparently means "breaking off" this worldline at $r_{g}$. In fact, in the general relativistic collapse, the worldline $r(R, \tau)$ does not break off, but asymptotically approaching $r_{g}$ for the entire life of the frozen star in the real external world into which it is embedded. Moreover, each event on the asymptotic worldline $r(R, t) \rightarrow r_{g}$ is an event on the worldline $r(R, \tau) \rightarrow r_{g}$. The 
absence of this phenomenon in the Newtonian theory gave rise a myth about breaking of the worldline $r(R, \tau)^{1}$.

\subsection{Freezing of the inner region and lack of horizon and singularity}

Inside the thin dust shell $r<r_{b}$, the space is flat and uniform $g_{11}=-1$. The temporal component of the metric on the shell $r=r_{b}$ is matched with the external Schwarzschild metric $g_{00}\left[r_{b}(t)\right]=1-r_{g} / r_{b}(t)$ and therefore inside, due to homogeneity, it is equal at any point to the same value $g_{00}[r(t)]=g_{00}\left[r_{b}(t)\right]$.

Since at any moment $t<\infty$ the shell is outside the gravitational radius $r_{b}(t)>r_{g}$, and the internal space is flat, neither the event horizon nor the singularity in the center are formed at the collapse of the thin dust shell.

Free test particles inside the shell move rectilinearly, but their movement begins to slow down due to the time dilation, and they quickly freeze where they were before the shell freezes.

Thus, the collapse of a thin dust shell leads to the formation of a hollow frozar, an object completely frozen in its own strong gravitational field. Such a frozar consists of a shell frozen over the gravitational radius and the empty interior with a flat spatial metric and frozen test particles.

It is important that the gravitational time dilation of $\tau$ w.r.t. $t$, which is absent in the Newtonian theory, turned out to be the internal mechanism stopping the general relativistic collapse of the shell without additional forces. In this case, there is also a kinematical time dilation due to the particle velocity.

It is also important to demonstrate in this extremely simple and obvious example of the fatal error of the naive extrapolation of the Newtonian scenario of collapse to general relativity, as a result of which earlier even for such a shell a transition to such a mythical state as a black hole with a horizon and a singularity was predicted.

In fact, as shown above, according to general relativity, the shell passes into the state of a hollow frozar, i.e. freezes over its gravitational radius, and its inner region, remaining be flat, become a place where nothing happens in terms of $t$, the external world's time.

\subsection{Frozar formation is independent on the choice of coordinates}

At the shell collapse, the conclusion about the formation of a hollow frozar instead of a black hole is not related with the particular choice of the Schwarzschild coordinates $r, t$ only, but remains valid for any other choice of coordinates.

Notice that the Schwarzschild's coordinates $r, t$ are physical coordinates in a static field that adequately take into account field symmetries and they are measured by standard scales along circles and world time clocks running synchronously everywhere. Therefore, the introduction of other parameters $x^{0}(t, r)$ and $x^{1}(t, r)$ instead of them does not change the physical results, since the description of physical processes and the structure of objects

\footnotetext{
${ }^{1}$ This is another evidence of the unfortunate fact for the specialists in mathematical physics that a formal knowledge of the mathematics of general relativity does not guarantee an understanding of its physical aspects and therefore can lead to such obvious errors in its applications to astrophysics.
} 
ultimately comes down to measuring the same really measurable physical coordinates of the particles. New coordinates, usually just some parameters that are often impossible to measure, and in order to obtain physically measurable quantities, one has to return to really measurable physical coordinates, the simplest of which are precisely the Schwarzschild coordinates $r, t$

This is clear from many attempts to replace $r$ by another radial coordinate in order to eliminate the singularity at $r=r_{g}$. Formally, for this it is enough to shift $r$ by $r_{g}$, i.e. $r=\bar{r}+r_{g}$, after which the line element (2) takes the form:

$$
d s^{2}=\frac{d t^{2}}{1+r_{g} / \bar{r}}-\left(1+\frac{r_{g}}{\bar{r}}\right) d \bar{r}^{2}-\left(\bar{r}+r_{g}\right)^{2} d \Omega^{2} .
$$

Here in the radial and temporal components of the metric there is only a singularity at $\bar{r}=0$, which is similar to the Newtonian theory case and seems more acceptable. However, now the angular line element $d \Omega^{2}$ appears as multiplied to $\left(\bar{r}+r_{g}\right)^{2}$.

But since the field of a static spherical source does not affect the standard scales along circles, on 2-spheres the physical coordinates remain the same as in flat spacetime. Therefore, if we want to measure $\bar{r}$ along circles, $\bar{r}+r_{g}$ should be expressed through the natural physical scale equal to the length of the circle divided to $2 \pi$ and we again return to the previous physical radial coordinate $r$.

Thus, the attempts to eliminate of singularities by a simple transformation of the radial coordinate turned out to be only a mathematical exercise, which do not change both the relationship between the really measured quantities and the physical consequences of the theory.

The situation is similar in other proposals to replace $r$ by another parameter, in particular, with the often popularized "tortoise" coordinate

$$
r^{*}=r+r_{g} \ln \left(\frac{r}{r_{g}}-1\right), \quad d r^{*}=\frac{d r}{1-r_{g} / r} .
$$

As soon as we turn to the physical aspects of the problem, and therefore to the real determination of the location of the event with the parameter $r^{*}$, we need to indicate a circle around the source where this event occurred, as well as the fact that $2 \pi r$ of standard scales are placed on this circle, which gives its physical length. In particular, an event on an unremarkable circle with $r=2 r_{g}$ corresponds to a parameter $r^{*}=r_{g}$, and at $r \rightarrow r_{g}$ will $r^{*} \rightarrow-\infty$. This shows that $r^{*}$ there is only a formal parameterization of the physical coordinate on the circle $r$ and does not have a direct physical meaning.

The situation is the same with the replacement $d \bar{\tau}^{2}=\left(1-r_{g} / r\right) d t^{2}$, relating different $\Delta \bar{\tau}$ by the identical intervals $\Delta t$. Moments of the local proper time $\bar{\tau}$ corresponding to the events on the circles $r$ acquire the meaning of a global time coordinate only after their connection with the globally defined moments of world time $t$.

The mentioned in the Introduction mathematical extrapolation to nonphysical region $t>\infty$ and $\tau>\tau_{g}$ was brought into a more "plain" form by formal transformation of the 
Schwarzschild physical coordinates $(t, r) \rightarrow\left(x^{0^{\prime}}, x^{1^{\prime}}\right)$. New coordinates were introduced so that the real relationships of the measured physical coordinates, expressing the properties of the real gravitational field of the source, were formally hidden. Such formal mathematical manipulations include, in particular, the Eddington-Finkelstein and Kruskal coordinates.

Since in all these cases the new coordinates are just a parameterization of the same Schwarzschild physical coordinates $(t, r)$ with only a corresponding change in the metric

$$
\begin{aligned}
& d t=\frac{\partial t}{\partial x^{\prime 0}} d x^{\prime 0}+\frac{\partial t}{\partial x^{\prime 1}} d x^{\prime 1}, \quad d r=\frac{\partial r}{\partial x^{\prime 0}} d x^{\prime 0}+\frac{\partial r}{\partial x^{\prime 1}} d x^{\prime 1}, \\
& g_{00}=\frac{\partial x^{\prime i}}{\partial t} \frac{\partial x^{\prime k}}{\partial t} g_{i^{\prime} k^{\prime}}, \quad g_{11}=\frac{\partial x^{\prime i}}{\partial r} \frac{\partial x^{\prime k}}{\partial r} g_{i^{\prime} k^{\prime}},
\end{aligned}
$$

then this does not change the proper time of the particle on the shell:

$$
\begin{aligned}
d \tau^{2} & =g_{00} d t^{2}+g_{11} d r^{2}-r^{2} d \Omega^{2}= \\
& =g_{0^{\prime} 0^{\prime}}\left(d x^{0^{\prime}}\right)^{2}+g_{1^{\prime} 1^{\prime}}\left(d x^{1^{\prime}}\right)^{2}+2 g_{0^{\prime} 1^{\prime}} d x^{0^{\prime}} d x^{1^{\prime}}-r^{2} d \Omega^{2} .
\end{aligned}
$$

Therefore, if this invariant proper time freezes w.r.t. the same invariant proper time of the static observer at a sufficient distance from the shell $\left(\sim 100 r_{g}\right)$, then the fact of freezing the proper time of the shell relative w.r.t. the proper time of the distant observer will remain the same in the new coordinates.

The statements, that the shell apparently "reaches" and "crosses" $r_{g}$ in the new coordinates, and then "falls" to the center, are erroneous, since they are based on ignoring the dilation of $\tau$ with respect to $t$ at $r \rightarrow r_{g}$. This was done by separating the local description of real physical objects and processes with them from the uniformly flowing time $t$ of the surrounding real world, which is unacceptable in the framework of GR.

The formal mathematical nature of these manipulations also reveals in the fact that the Schwarzschild physical coordinates $(t, r)$ are directly measured, while the procedure for measuring the usually introduced parameterizations $x^{0}, x^{1}$ is never specified.

Inside and around the shell, GR does not give the right to ignore the global simultaneity of events in the rest frame of the shell center. Outside and on the shell, where the field is the Schwarzschild one, the simultaneity of events is established by Einstein's synchronization, as in other static fields. Therefore, both inside and outside the shell, Minkowski's definition that "the world is a set of simultaneous events" remains valid.

This imposes a constraint on the local proper times $\tau=\tau(R, t)$, which during the evolution of the external world in the physical region $t \in\left[t_{0}, \infty\right)$ reduces the physical region of change of $\tau$ to a finite interval $\tau \in\left[0, \tau_{g}\right)$. This fact of the dilation of the local proper times w.r.t. $t$ plays a key role for understanding the process and the result of relativistic collapse not only in the case of a shell, but for more complicated compact objects also, which will be considered below. 


\section{Exact solutions for a spherical homogeneous dust star}

\subsection{Friedmann-Tolman solution in comoving coordinates}

To obtain the exact solution of the Einstein equations inside a spherical dust star in locally comoving frames, spherical symmetry and zero pressure condition are sufficient. The Friedmann solution for a locally homogeneous density $\rho=\rho(\tau)$ and later a Tolman's general solution for an arbitrary density $\rho=\rho(r, \tau)$ allow to obtain expressions for worldlines of the dust particles in all layers [2,12].

In the comoving frame, the radial coordinate of each layer $r(\tau)$ remains fixed and equal to the value $R$ at the initial moment $\tau=0$. Inside a dust star $R \leq R_{b}$, where $R_{b}$ is an initial radial coordinate of the surface, the line element in the comoving coordinates can therefore be represented as:

$$
d s^{2}=c^{2} d \tau^{2}-e^{\lambda(\tau, R)} d R^{2}-r^{2}(R, \tau) d \Omega^{2}
$$

Tolman's solution for parabolic velocity reduces to expressions (see. [12]):

$$
e^{\lambda}=r^{\prime 2}, \quad \dot{r}^{2}=\frac{F}{r}, 8 \pi G \rho=\frac{F^{\prime}}{r^{\prime} r^{2}} .
$$

Here $F(R)$ is an unknown function depending on the choice of density distribution.

Integration in (25) gives

$$
\tau= \pm \frac{1}{F^{1 / 2}} \int d r r^{3 / 2},
$$

where $F(R)=r_{g}$ at $R \geq R_{b}$. Thus, the expressions for the worldline of particles in a given stellar layer $\tau(R, r)$ and $r(R, \tau)$ follow from (25) - (26):

$$
\tau=\frac{2}{3 F^{1 / 2}}\left(R^{3 / 2}-r^{3 / 2}\right), \quad r=\left(R^{3 / 2}-\frac{3 F^{1 / 2}}{2} \tau\right)^{2 / 3} .
$$

The simplest additional condition for determining the form of the function $F(R)$ is the condition of local homogeneity, i.e. independence of density from spatial coordinates $\rho=\rho(\tau)$, at the same values of the local proper time $\tau(R, r)$ (the "same-age" events).

To simplify, it is required not only the choice of the initial locally homogeneous distribution at $\tau=0$, but also the choice of those initial velocities of falling at which this uniformity will be maintained in later moments. There are three such regimes of local velocity - parabolic $(k=0)$, when the initial velocity is zero at infinity, elliptical $(k=1)$ and hyperbolic $(k=-1)$, when the initial velocity at $r=R$ is equal to zero or $-\left(2 r_{g} / R\right)^{1 / 2}$, respectively. In this first paper, we consider the simplest parabolic case only ( $k=0$ ), and two other velocities $(k= \pm 1)$ will be considered in the second paper [7].

To find $F$, from (25) and the homogeneity condition we obtain the following relationships $(\kappa=8 \pi G / 3)$ :

$$
F^{\prime}=3 \kappa \rho(\tau) r^{2} r^{\prime}=\kappa \rho(\tau)\left(r^{3}\right)^{\prime},
$$




$$
F=\kappa \rho(\tau) r^{3}=\kappa \rho(0) R^{3}=\kappa \rho(0) R_{b}^{3} \frac{R^{3}}{R_{b}^{3}} .
$$

Then, taking into account that $r_{g}=2 G M=\kappa \rho(0) R_{b}^{3}$, we arrive at the expressions

$$
F(R)=r_{g} \frac{R^{3}}{R_{b}^{3}} \equiv r_{g, R}, \quad \rho(\tau)=\rho(0) \frac{R^{3}}{r^{3}} .
$$

Using this expression for $F$, we can calculate from (27)-(29) not only $\dot{r}$, but $r^{\prime}$ also. At first, from (28)-(30) we obtain:

$$
F^{\prime}=\kappa \rho(\tau) r^{2} r^{\prime}=\kappa \rho(0) R^{3} r^{\prime}=3 r_{g} \frac{R^{3}}{r R_{b}^{3}} r^{\prime},
$$

and then, comparing with $F^{\prime}=3 R^{2} r_{g} / R_{b}^{3}$, following from (30), we obtain

$$
r^{\prime}=\frac{r}{R}
$$

At the introduction of parameterization:

$$
r=a \chi, \quad R=a_{0} \chi, \quad r_{b}=a \chi_{b}, \quad R_{b}=a_{0} \chi_{b},
$$

where $a=a(\tau), a_{0}=a(0)$, the line element (24) takes the standard form, in which the Friedmann solution is usually represented [12]:

$$
d s^{2}=c^{2} d \tau^{2}-a^{2}\left(d \chi^{2}+\chi^{2} d \Omega^{2}\right)
$$

The expression for the local velocity (25) goes over to the evolution equation for $a$ (Friedmann equation) for the parabolic velocity:

$$
\dot{a}^{2}=\frac{a_{m}}{a} .
$$

\subsection{Transformation of the solutions from comoving to static coordinates}

Following the OS [1], inside the star, we transform the Tolman solution (24) in terms of $R, \tau$ to a solution in terms of $r, t$. This is necessary both for determining the instantaneous structure of the star on the hypersurface of simultaneity $t=$ const., and for matching on the surface with the external static metric (2).

The transition from $d \tau, d R$ to $d t, d r$ is given by the general transformations:

$$
d x^{i}=\frac{\partial x^{i}}{\partial x^{\prime k}} d x^{\prime k}
$$

which take the form:

$$
d t=\dot{t} d \tau+t^{\prime} d R, \quad d r=\dot{r} d \tau+r^{\prime} d R .
$$

The new line element, transformed from (24), is non-diagonal in general case. However, the metric must be diagonal, at least on the surface, in order to be matched with the external metric (2). Therefore, we search for the simplest exact solution in terms of $r, t$ in the form of a diagonal metric.

Notice that the instantaneous structure of a star as an extended object must be defined on the global hypersurface of simultaneity, coinciding on its surface with the hypersurface 
$t=$ const . This also assumes the presence of synchronized clocks in the entire volume of the star, showing the same moment of world time $t$. This is easily implemented for the diagonal metric, and can be very difficult for the non-diagonal case.

Thus, we proceed from the diagonal line element:

$$
d s^{2}=e^{v(r, t)} d t^{2}-e^{\lambda(r, t)} d r^{2}-r^{2} d^{2} \Omega, \quad r \leq r_{b},
$$

with the matching condition $e^{v\left(r_{b}, t\right)}=e^{-\lambda\left(r_{b}, t\right)}=1-r_{g} / r_{b}$. Substituting (37) into (38) gives:

$$
d s^{2}=\left(e^{v} \dot{t}^{2}-e^{\lambda} \dot{r}^{2}\right) d \tau^{2}-\left(e^{\lambda} r^{\prime 2}-e^{v} t^{\prime 2}\right) d R^{2}-r^{2} d^{2} \Omega+2\left(e^{v} \dot{t} t^{\prime}-e^{\lambda} \dot{r} r^{\prime}\right) d \tau d R .
$$

Using (24)-(25), we obtain from (38)-(39):

$$
\begin{gathered}
g_{00}(\tau, R)=e^{v} \dot{t}^{2}-e^{\lambda} \dot{r}^{2}=1, \quad g_{11}(\tau, R)=-\left(e^{\lambda} r^{\prime 2}-e^{v} t^{\prime 2}\right)=-\bar{r}^{\prime 2}, \\
g_{01}(\tau, R)=e^{v} \dot{t} t^{\prime}-e^{\lambda} \dot{r} r^{\prime}=0 .
\end{gathered}
$$

The relations (40) allow us to express the components of the metric:

$$
e^{\lambda}=\frac{t^{\prime 2}+\dot{t}^{2} \bar{r}^{\prime 2}}{\dot{t}^{2} r^{\prime 2}-t^{\prime 2} \dot{r}^{2}}, \quad e^{v}=\frac{r^{\prime 2}+\bar{r}^{\prime 2} \dot{r}^{2}}{\dot{t}^{2} r^{\prime 2}-t^{\prime 2} \dot{r}^{2}}
$$

The Eq. (41), expressing the diagonality of metric, at inserting (42), takes the form:

$$
g_{01}=\frac{\dot{t} t^{\prime}\left(r^{\prime 2}+\bar{r}^{\prime 2} \dot{r}^{2}\right)-\left(t^{\prime 2}+\dot{t}^{2} \bar{r}^{\prime 2}\right) \dot{r} r^{\prime}}{\dot{t}^{2} r^{\prime 2}-t^{\prime 2} \dot{r}^{2}}=0
$$

By rewriting this condition in the form of an equation for $t^{\prime}$ :

$$
t^{\prime 2} \dot{r}-t^{\prime} \dot{t} r^{\prime}\left(1+\dot{r}^{2}\right)+\dot{t}^{2} \dot{r} \bar{r}^{\prime 2}=0,
$$

we get two solutions:

$$
t^{\prime}=\frac{\dot{t} r^{\prime}}{2 \dot{r}}\left[\left(1+\dot{r}^{2}\right) \pm\left(1-\dot{r}^{2}\right)\right] .
$$

The case of the plus sign gives $t^{\prime}=\dot{t}^{\prime} / \dot{r}$, which is non-physical, since $t^{\prime}$ diverges at $\dot{r} \rightarrow 0$. The minus sign gives the desired diagonality condition for the metric:

$$
\frac{t^{\prime}}{\dot{t}}=\dot{r} r^{\prime} .
$$

After exclusion of $\dot{r} \rightarrow 0$ by using (46), the metric in (42) is simplified:

$$
e^{\lambda}=\frac{1}{1-F / r}, \quad e^{v}=\frac{1}{\dot{t}^{2}(1-F / r)} .
$$

\subsection{Oppenheimer-Snyder solution for a uniform dust star}

The diagonality condition (46) includes $r^{\prime}$, which is known from (32), while the expressions for $\tau(r, R), r(\tau, R)$ and $\dot{r}$ follow from (27) and (30):

$$
\tau=\frac{2}{3 r_{g, R}^{1 / 2}}\left(R^{3 / 2}-r^{3 / 2}\right),
$$




$$
r(R, \tau)=R\left(1-\frac{3 r_{g, R}^{1 / 2}}{2 R_{b}^{3 / 2}} \tau\right)^{2 / 3}, \quad \dot{r}=-\frac{r_{g, R}^{1 / 2}}{r^{1 / 2}} .
$$

To determine the worldline $r(t, R)$ in the OC method [1], we must first introduce an auxiliary function $y(R, r)$ through the relationships:

$$
t=M(y), \quad \dot{t}=\frac{\partial t}{\partial y} \dot{y}, \quad t^{\prime}=\frac{\partial t}{\partial y} y^{\prime},
$$

where the function $M$ can be find from matching with the external metric. The diagonality condition (46), as a result, takes the form of an equation for $y$ :

$$
\frac{t^{\prime}}{\dot{t}}=\frac{y^{\prime}}{\dot{y}}=\dot{r} r^{\prime}=-\frac{\left(r_{g, R} r\right)^{1 / 2}}{R} \text {. }
$$

The second step of the method is to solve Eq. (51) for $y$. In the OS paper [1] this solution has been taken as:

$$
y(R, r)=\frac{1}{2}\left(\frac{R^{2}}{R_{b}^{2}}-1\right)+\frac{R_{b}}{R r_{g}} r .
$$

Its derivation has been presented in [3-4] and, in the general case of three velocities is presented in [7].

The third step is to determine the value of $y$ on the surface $y\left(R_{b}, r\right)=r / r_{g}$, where $M\left(y_{b}\right)$ should coincide with the right-hand side of (13). This allows us to identify $M\left(y_{b}\right)$ with $t\left(r_{g} y, R_{b}\right)$ in (13). Thus, the replacements $R \rightarrow R_{b}, r \rightarrow r_{g} y$ in (13) give us the OS solution for the internal worldlines:

$$
t(R, r)=r_{g}\left[\frac{2}{3}\left(\frac{R_{b}^{3 / 2}}{r_{g}^{3 / 2}}-y^{3 / 2}\right)-2 y^{1 / 2}+\ln \frac{y^{1 / 2}+1}{y^{1 / 2}-1}\right] .
$$

For $\dot{t}$ and $t^{\prime}$ this formula, using $y^{\prime}=R / R_{b}^{2}$ and $\dot{y}=-\left(R / r r_{g} R_{b}\right)^{1 / 2}$, gives:

$$
\dot{t}=-\frac{r_{g} y^{3 / 2} \dot{y}}{y-1}=\left(\frac{r_{g} R}{R_{b} r}\right)^{1 / 2} \frac{y^{3 / 2}}{y-1}, \quad t^{\prime}=-\frac{r_{g} y^{3 / 2} y^{\prime}}{y-1}=-\frac{r_{g} R}{R_{b}^{2}} \frac{y^{3 / 2}}{y-1},
$$

and the diagonality condition (51) is satisfied.

As a result, the metric in (47) takes the form

$$
e^{\lambda}=\frac{1}{1-r_{g, R} / r}, \quad e^{v}=\frac{1}{\dot{t}^{2}\left(1-r_{g, R} / r\right)},
$$

and, after insertion of $\dot{t}$ from (54), $e^{v}$ takes the final form:

$$
e^{v(r, t)}=\frac{1}{1-r_{g, R} / r} \cdot \frac{\left[1-\left(3-R^{2} / R_{b}^{2}\right) r_{g} R / 2 r R_{b}\right]^{2}}{\left[1-\left(1-R^{2} / R_{b}^{2}\right) r_{g} R / 2 r R_{b}\right]^{3}} .
$$




\subsection{Worldlines of particles in the layers and their asymptotes}

The diagrams of worldlines $r(R, t)$ of dust particles inside the dust star for the parabolic OS solution (52)-(53) are shown in Figs. 2-3. Fig. 2 shows the asymptotes $r_{\infty}(R)$ to which the worldlines $r(R, t)$ of particles in the layers tend at $t \rightarrow \infty$. The asymptotes, parallel to the worldline of the center, are located almost equidistant, slightly condensing near the surface.

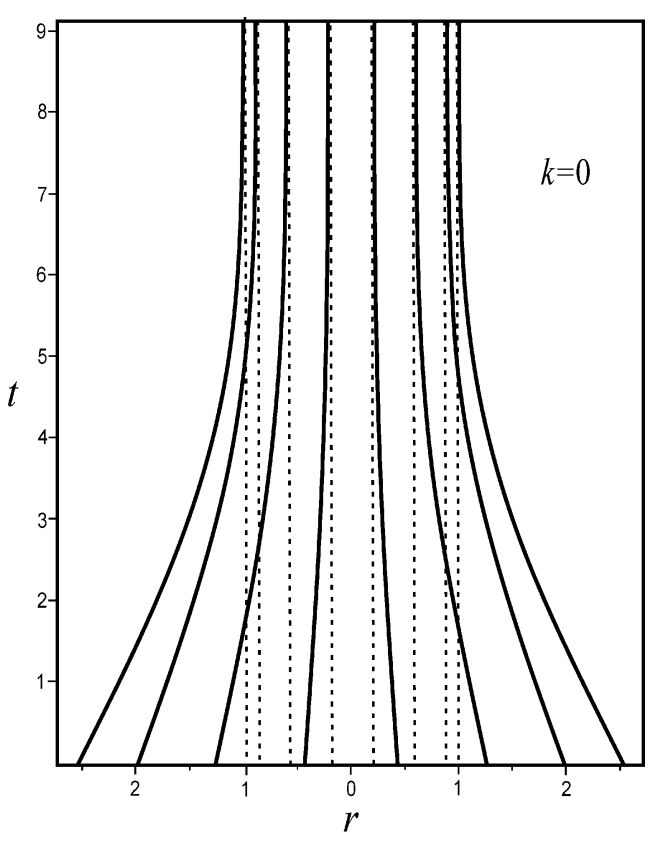

Fig. 2. Worldlines of star particles along the diameter. $R_{b}=3.5$ at $\tau=0$ (in units $r_{g}$ ) by OS decision (52)-(53). At large $t$, the worldlines are timelike, the surface freezes outside $r_{g}$, the inner layers freeze near their asymptotes $r_{\infty}(R)$ (dashed line) from (60).

At $t \gg r_{g}$ the surface asymptotically approaches the gravitational radius $r_{b}(t) \rightarrow r_{g}$ and the logarithmic term dominates in (8), which leads to:

$$
t \simeq \frac{2 R_{b}^{3 / 2}}{3 r_{g}^{1 / 2}}-\frac{8}{3} r_{g}-r_{g} \ln \left(\frac{r_{b}^{1 / 2}-r_{g}^{1 / 2}}{r_{b}^{1 / 2}+r_{g}^{1 / 2}}\right)
$$

This gives a formula for the asymptotes of the surface:

$$
r_{b}(t) \simeq r_{g}\left(1+a e^{-t / r_{g}}\right)>r_{g}, \quad a \equiv 4 \exp \left(\frac{2 R_{b}^{3 / 2}}{3 r_{g}^{3 / 2}}-\frac{8}{3}\right) \text {. }
$$

At $t \gg r_{g}$ the logarithmic term in (53) also dominates in the internal layers and we have $y \rightarrow 1$. This leads to the equation for the asymptotes $r(R, t) \rightarrow r_{\infty}(R)$ : 


$$
\left.y(R, t)\right|_{t \rightarrow \infty} \rightarrow \frac{1}{2}\left(\frac{R^{2}}{R_{b}^{2}}-1\right)+\frac{R_{b}}{r_{g} R} r_{\infty}(R)=1 .
$$

The expression for the asymptotes, therefore, has the form:

$$
r_{\infty}(R)=R \frac{3 r_{g}}{2 R_{b}}\left(1-\frac{R^{2}}{3 R_{b}^{2}}\right),
$$

At $R=R_{b}$ the Eq. (60) gives $r_{\infty}\left(R_{b}\right)=r_{g}$. Rewriting this formula as:

$$
r_{\infty}(R)=r_{g, R}+\frac{3 r_{g, R}}{2}\left(\frac{R_{b}^{2}}{R^{2}}-1\right)
$$

we see that $r_{\infty}(R)$ represents an "internal" gravitational radius of the layer $R$, formed by the effective gravitational radius $r_{g, R}$ of the matter inside and the contribution of the outer layers to the time dilation described by the second term of (61).

Formulas for the asymptotes (60)-(61) follow from (56) also at taking into account the freezing time at $\exp \left[v\left(r_{\infty}\right)\right]=0$, when the time component of the metric vanishes:

$$
1-\left(3-R^{2} / R_{b}^{2}\right) \frac{r_{g} R}{2 r_{\infty} R_{b}}=0 .
$$

As it follows from (60), the asymptotes of the inner layers are located almost equidistantly $\sim R$ as close to the center (Fig. 2), and only layers near the surface become denser due to spatial compressions and later freezing, which is described by the factor $-r_{g} R^{3} / 2 R_{b}^{3}$. The proper time on each layer freezes at $\tau_{\infty}(R)$, the value of which we find by substituting $r_{\infty}(R)$ into (48):

$$
\begin{gathered}
\tau_{\infty}(R)=\frac{2 R_{b}^{3 / 2}}{3 r_{g}^{1 / 2}}-\frac{3^{1 / 2} r_{g}}{2^{1 / 2}}\left(1-\frac{R^{2}}{3 R_{b}^{2}}\right)^{3 / 2}, \\
\tau_{\infty}(0)=\frac{2 r_{g}}{3}\left(\frac{R_{b}^{3 / 2}}{r_{g}^{3 / 2}}-\frac{3^{3 / 2}}{2^{3 / 2}}\right), \quad \tau_{\infty}\left(R_{b}\right)=\frac{2 r_{g}}{3}\left(\frac{R_{b}^{3 / 2}}{r_{g}^{3 / 2}}-1\right) .
\end{gathered}
$$

This shows that the center freezes before the all layers at the moment $\tau_{\infty}(0)$, and the surface freezes after all the layers at the moment $\tau_{\infty}\left(R_{b}\right)$. Thus, the time interval during which all layers solidify is equal to the difference between the moments of freezing of the surface and center:

$$
\Delta \tau_{\infty} \equiv \tau_{\infty}\left(R_{b}\right)-\tau_{\infty}(0)=\frac{2}{3}\left(\frac{3^{3 / 2}}{2^{3 / 2}}-1\right) r_{g} \approx 0.558 r_{g} .
$$

For a star of mass $10 M_{\circ}$ and $r_{g} \simeq 30 \mathrm{~km}$, the difference between the moments shown by the frozen standard clocks on the surface and in the center is about 6 microseconds. The interval of world time between the freezing of the center and the surface, of course, is much larger. 
Consider the dependence on $t$ of the main variables at large $t$. Asymptotic form (53) for $t \gg r_{g}$ and $y \rightarrow 1$ :

$$
t \simeq r_{g} \ln a-r_{g} \ln \frac{y-1}{4}, \quad a=\exp \left(\frac{2 R_{b}^{3 / 2}}{3 r_{g}^{3 / 2}}-\frac{8}{3}\right),
$$

gives an asymptotic expression for the worldline $r(R, t)$ :

$$
r(R, t) \simeq R \frac{3 r_{g}}{2 R_{b}}\left(1-\frac{R^{2}}{3 R_{b}^{2}}+a e^{-t / r_{g}}\right) .
$$

Substituting this into (27), we find the dependence of the moments of proper time $\tau(R, t)$ from $t$ inside the star:

$$
\tau(R, t)=\frac{2 R_{b}^{3 / 2}}{3 r_{g}^{1 / 2}}-\frac{3^{1 / 2} r_{g}}{2^{1 / 2}}\left(1-\frac{R^{2}}{3 R_{b}^{2}}+a e^{-t / r_{g}}\right)^{3 / 2} .
$$

At $t \rightarrow \infty$ this expression turns into (63). For center and surface we have:

$$
\begin{gathered}
\tau(0, t)=\frac{2 R_{b}^{3 / 2}}{3 r_{g}^{1 / 2}}-\frac{3^{1 / 2} r_{g}}{2^{1 / 2}}\left(1+a e^{-t / r_{g}}\right)^{3 / 2} . \\
\tau\left(R_{b}, t\right)=\frac{2 R_{b}^{3 / 2}}{3 r_{g}^{1 / 2}}-\frac{3^{1 / 2} r_{g}}{2^{1 / 2}}\left(\frac{2}{3}+a e^{-t / r_{g}}\right)^{3 / 2} .
\end{gathered}
$$

and for their difference we obtain:

$$
\begin{aligned}
& \frac{1}{r_{g}}\left[\tau\left(R_{b}, t\right)-\tau(0, t)\right]=\frac{3^{1 / 2}}{2^{1 / 2}}\left(1+a e^{-t / r_{g}}\right)^{3 / 2}-\frac{2}{3}\left(1+\frac{3}{2} a e^{-t / r_{g}}\right)^{3 / 2} \simeq \\
& \simeq \frac{2}{3}\left(\frac{3^{3 / 2}}{2^{3 / 2}}-1\right)+\frac{3}{2}\left(\frac{3^{1 / 2}}{2^{1 / 2}}-1\right) a e^{-t / r_{g}}
\end{aligned}
$$

\section{Freezing at the collapse of charged and rotating stars}

\subsection{Collapse of a charged spherical star}

For the charged spherical dust star, the metrics outside and on the surface are given by the Reissner-Nordström solution:

$$
g_{00}(r)=-g_{11}^{-1}(r)=1-\frac{r_{g}}{r}+\frac{G Q^{2}}{r^{2}}, \quad r \geq r_{b} .
$$

Since the entire electric charge of the star is concentrated on its surface, the inner layers remain neutral. The mass of the charged surface layer is very small, so that the collapse of the internal neutral matter occurs as in a dust star and is described by the OS solution considered in the previous Section, slightly corrected by the contribution of the electric field energy.

In this case the thin charged surface layer does not fall freely in the gravitational field of the star, but is decelerates by its own electric field and lag behind free falling inner neutral layers. As a result, a charged dust star will consist of a neutral core, which will 
quickly turn into a locally homogeneous frozar, and a thin charged dust shell hanging above it at a certain distance.

Some observational effects associated with the later falling of the charged surface layer, decelerated by own electric field, will be discussed in the Section 5.5.

\subsection{Collapse of rotating star}

The space-time interval between two events around and on the surface of a rotating star is given by the Kerr solution, which in the Boyer-Lindquist coordinates has the form $($ see $[12,13])$ :

$$
\begin{aligned}
& d s^{2}=g_{00} d t^{2}-\frac{\rho^{2}}{\Delta} d r^{2}+\frac{2 r_{g} r a}{\rho^{2}} \sin ^{2} \theta d \varphi d t- \\
& -\left(r^{2}+a^{2}+\frac{r_{g} r a^{2}}{\rho^{2}} \sin ^{2} \theta\right) \sin ^{2} \theta d \varphi^{2}-\rho^{2} d \theta^{2} .
\end{aligned}
$$

Here $\rho^{2}=r^{2}+a^{2} \cos ^{2} \theta, \Delta=r^{2}+a^{2}-r_{g} r$ and $g_{00}=1-r_{g} r / \rho^{2}$. At $a \rightarrow 0$ these coordinates turn to the Schwarzschild coordinates. The limiting case $g_{00} \rightarrow 0$ at $\rho^{2} \rightarrow r_{g} r$ corresponds to $r \rightarrow r_{0}$ with

$$
r_{0}=\frac{r_{g}}{2}\left[1+\left(1-\frac{4 a^{2}}{r_{g}^{2}} \cos ^{2} \theta\right)^{1 / 2}\right]
$$

The proper time of a particle resting at $(r, \theta, \varphi)=$ const. is defined as:

$$
d \tau^{2}=g_{00} d t^{2} .
$$

At $r \rightarrow r_{0}$ this proper time freezes w.r.t. $t$, which is a purely gravitational time dilation.

The proper time of the comoving to the surface particle is slowed down in terms of $t$ both gravitationally and kinematically:

$$
\begin{gathered}
d s^{2}=g_{00} d t^{2}\left(1-\mathbf{v}^{2}\right), \\
\mathbf{v}^{2}=g_{00}^{-1}\left\{\frac{\rho^{2}}{\Delta} v_{r}^{2}+\rho^{2} v_{\theta}^{2}+\left[\left(r^{2}+a^{2}+\frac{r_{g} r a^{2}}{\rho^{2}} \sin ^{2} \theta\right) \Omega+\frac{2 r_{g} r a}{\rho^{2}}\right] \Omega \sin ^{2} \theta\right\},
\end{gathered}
$$

where $v_{r}=d r / d t, v_{\theta}=d \theta / d t, v_{\varphi}=d \varphi / d t=\Omega$. On the equatorial plane $\theta=\pi / 2$, where $\rho=r$, these expressions take the form:

$$
\begin{gathered}
d \tau^{2}=d t^{2}\left(1-\frac{r_{g}}{r}\right)\left(1-\mathbf{v}^{2}\right), \\
\mathbf{v}^{2}=\left(1-\frac{r_{g}}{r}\right)^{-1}\left[\frac{r^{2}}{a^{2}} v_{r}^{2}+\left(r^{2}+a^{2}+\frac{a^{2} r_{g}}{r}\right) \Omega^{2}-\frac{2 r_{g} a}{r} \Omega\right] .
\end{gathered}
$$

At $\mathbf{v}^{2} \rightarrow 1$ the Eq. (79) gives: 


$$
\frac{r^{2} v_{r}^{2}}{a^{2}}-\frac{2 r_{g} a}{r} \Omega+\left(r^{2}+a^{2}+\frac{a^{2} r_{g}}{r}\right) \Omega^{2} \rightarrow\left(1-\frac{r_{g}}{r}\right)
$$

Therefore, at $r \rightarrow r_{g}$ we have:

$$
\left(r_{g}^{2}+2 a^{2}\right) \Omega^{2}-2 a \Omega+\frac{r_{g}^{2}}{a^{2}} v_{r}^{2} \rightarrow 0 .
$$

Thus, at approaching $r_{0}$ the surface of a rotating star practically freezes in terms of $t$, the inner layers also freeze, and the star turns to a rotated frozar.

Gravitational time dilation in the star's layers (in the static frame), like a spherical star, is determined by the effective gravitational radius $\tilde{r}_{g, R}$ at the level of this layer. The latter depends both on the internal gravitational radius $r_{g, R}=r_{g} R^{3} / R_{b}^{3}$ and on the time dilation effect generated by matter outside this layer, described by the function $\beta(r, \theta)$ :

$$
d \tau^{2}=d t^{2}\left(1-\frac{r r_{g, R}}{\rho^{2}} \beta(r, \theta)\right)
$$

Notice that the angular momentum of a rotating star is conserved, and its external field remains the same as before freezing, since freezing does not mean an absolute stopping. The local velocity of particles on the frozar's surface is close to the light speed, and for the external static frame the frozar looks like a practically ellipsoidal star.

\section{Heterogeneity of structure and sticking together of frozars}

\subsection{Freezing of more than half of the frozar mass close to surface}

The radius $R_{M / 2}$, inside of which half the mass of a Newtonian homogeneous star is contained, can be find from the mass ratio:

$$
\frac{R_{b}^{3}}{R_{M / 2}^{3}}=\frac{M}{M / 2}=2, \quad R_{M / 2}=R_{b} / 2^{1 / 3} \simeq 0.7937 R_{b} .
$$

Thus, the second half the star's mass is contained on the surface layer having a thickness of about one fifth of the star's radius $0.2063 R_{b}$.

As T. Marshall firstly noticed [14], half of the mass of the relativistic dust star in the late stages of collapse is concentrated in the layer near the surface several times smaller in thickness than in the Newtonian case. In the framework of a certain analogue of the OS solution, studied in [14], it was obtained $\delta R \sim 0.03 R_{b}$ placed above $0.97 R_{b}$.

In [4], the value of this radius $R_{M / 2}$ was calculated in the case of the OS solution and it turned out that half of the star's mass, after freezing and transition to the frozar state, becomes contained on the surface layer of thickness $0.06 R_{b}$ above $0.97 R_{b}$. In view of the fact that this is a new relativistic effect that takes place for all compact stars, we present here the details of calculation.

For the layer $R=R_{M / 2}$ we obtain the value of the radius, inside which is half the mass of the frozar, the formula for asymptotes (60): 


$$
r_{\infty}\left(R_{M / 2}\right)=R_{M / 2} \frac{3 r_{g}}{2 R_{b}}\left(1-\frac{R_{M / 2}^{2}}{3 R_{b}^{2}}\right) .
$$

At the initial moment, a homogeneous dust star with a radius of the surface $R_{b} \gg r_{g}$ is almost Newtonian and therefore $R_{M / 2}$ still equal to $R_{M / 2}=R_{b} / 2^{1 / 3}$ as in (83). Substituting this value into (84), we find:

$$
r_{\infty}\left(R_{M / 2}\right)=\frac{3}{2^{4 / 3}}\left(1-\frac{1}{3 \cdot 2^{2 / 3}}\right) r_{g} \simeq 0,9406 r_{g} .
$$

Thus, this is a new effect of heterogeneity at $t=$ const. in the structure of locally homogeneous compact relativistic stars. As can be seen from (60) and Figs. 3-4, the asymptotes of the inner layers are almost equidistant in most of the stellar volume around the center $r_{a}(R) \sim R$. Only the layers initially close to the surface become denser, but such a concentration has a purely kinematical reason and occurs due to their later freezing and stronger radial contractions. These layers, however, remain locally homogeneous and fall freely in local frames.

For neutron and quark stars, this effect manifests itself in a less evident form, although it can be modeled and studied over time using new observational methods. In particular, if the structure of such stars is studied more accurately, this kind of the relativistic heterogeneity should be taken into account.

In the case of frozars, this effect is manifested in the largest possible form - half of the mass (or rather energy) of frozar is concentrated in the layers almost near the surface.

For rotating relativistic stars and their systems, this effect of compaction near the surface leads to additional observable effects due to the redistribution of the total angular momentum to the layers closer to the surface in the equatorial region. In particular, the rotation frequency of neutron stars will be less than that of a Newtonian star with the same mass and angular momentum.

\subsection{Formation of the accretionary landscape on the frozars}

The surfaces of white dwarfs and neutron stars do not contain large inhomogeneities due to their strong gravity. Any accreting matter is also absorbed by a compact star or distributed over its surface.

In the case of frozars, the situation is the opposite due to extremely strong gravity: the accreting matter, falling almost radially, quickly freeze on the surface, and the transverse size of the accreting part of the matter practically does not change. As a result, the matter becomes radially flattened without spreading over the surface.

This leads to an inhomogeneous distribution of the accreting matter on the surface of the frozar, similar to the concentrated masses (masons) on the moon. In disk accretion, the heterogeneity is maximal near the equator.

Of particular interest is the radial falling of a neutron star on a more massive frozar (Fig. 4). The matter of the neutron star is flattened and freeze on the surface of the frozar in the form of a local "hairstyle", a large mascon, which leads to a distinctive local inhomogeneity of the gravitational field of the frozar.

Thus, the formation of an inhomogeneous landscape on the surface of the frozar as a result of accretion distinguishes the frozars from two other classes of compact objects - 
white dwarfs and neutron stars. The observational effects of such a landscape, associated with local anomalies of the gravitational field of the frozar, are discussed in Section 5.2.

It is possible to use the degree of heterogeneity of the surface of frozars to determine their age, and this should be studied in the case of real objects, since such correlation is possible for some classes of objects. An explosion of a neutron star due to tangential contraction during the fall is also possible, which leads to the observable burst.

\subsection{Sticking together of frozars without merging}

The radial falling of ordinary matter onto the frozar (accretion) increases its mass, but only slightly changes the configuration of its gravitational field. However, at a radial falling of two or more frozars to a common center of inertia, there appear new phenomena.

In Newtonian gravity, the merger of two compact spherical objects can form a more massive compact object, the final stable state of which also become mainly spherical. In general relativity, two or more frozars cannot merge at approaching and freeze at a certain distance from each other, forming a frozen cluster of two or more frozars.

Let two frozars of equal mass $m_{1}=m_{2}=m$ and surface radius $r_{b}$, very close to their gravitational radii $r_{b}>r_{g}=2 G m$, radially fall to the common center of inertia, where the center of the static coordinate system with the radial coordinate $r$ and world time $t$ is located. The gravitational radius of the system is $\bar{r}_{g} \simeq 2 r_{g}$. At falling, the radial coordinate of the center of each frozar $r_{1}$ and $r_{2}$ remains larger $\bar{r}_{g}$, i.e. $r_{1}>\bar{r}_{g}$ and $r_{2}>\bar{r}_{g}$ due to the freezing of all processes, including the falling, in terms of $t$ (Fig. 5). Thus, in GR, two frozars cannot merge and it occurs their sticking together only. The same situation, even more obvious, holds for the three frozars (Fig. 5).

This fact is especially clear in the case of four radially falling frozars of the same mass located at the vertices of the tetrahedron. Here $\bar{r}_{g}=2 G M$, the gravitational radius of the system with $M \simeq 4 m$, about 4 times larger than the gravitational radius $r_{g}=2 G m$ of any of the frozars (Fig. 5). In fact, a frozen cluster forms a gravitational crystal, where the distances between the frozars are several times exceeds the size of each of them.

In the general case, the approach of $n$ frozars of equal mass, falling symmetrically to the common center of inertia, is similar to the collapse of the dust shell and therefore they freeze near the gravitational radius of the system

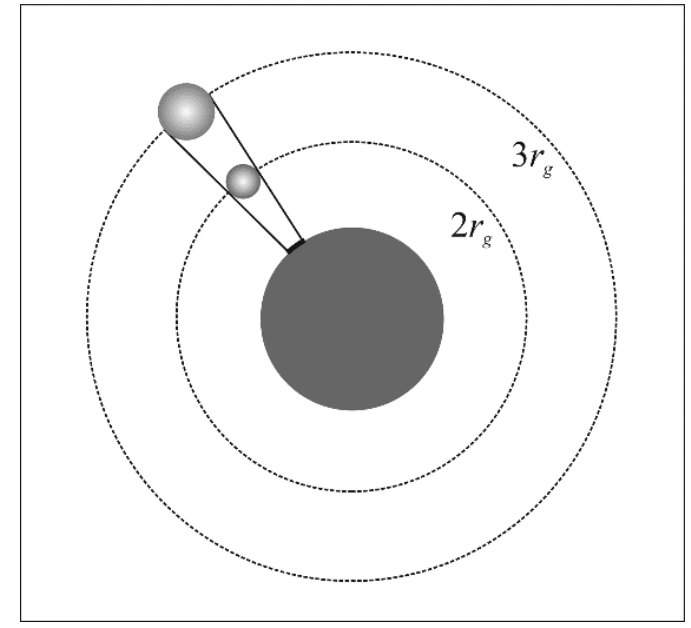

Fig. 4. Radial falling of a neutron star of mass $1.5 M_{\odot}$ on a frozar of mass $7 M_{\circ}$, leading to the flattening of the neutron star (like a mascon on the Moon) and freezing on the frozar's surface in the form of a "hairstyle". This leads to local inhomogeneity of the frozar field and its shadow. 
$\bar{r}_{g} \simeq n r_{g}$ due to the gravitational freezing of all processes in the rest frame of the system's center of inertia.

Notice that due to further accretion of ordinary matter the clusters of frozars become a cluster of frozars and ordinary matter, since the falling matter also freeze on the gravitational field of the cluster, if it had not time to fall onto one of the frozars and freeze on it. Thus, in astrophysics and cosmology, at modeling the structure of the most compact gravitationally frozen massive or supermassive clusters of frozars, in addition to ordinary processes, it is necessary to take into account the formation of gravitationally frozen inhomogeneous clusters of frozars and ordinary matter.
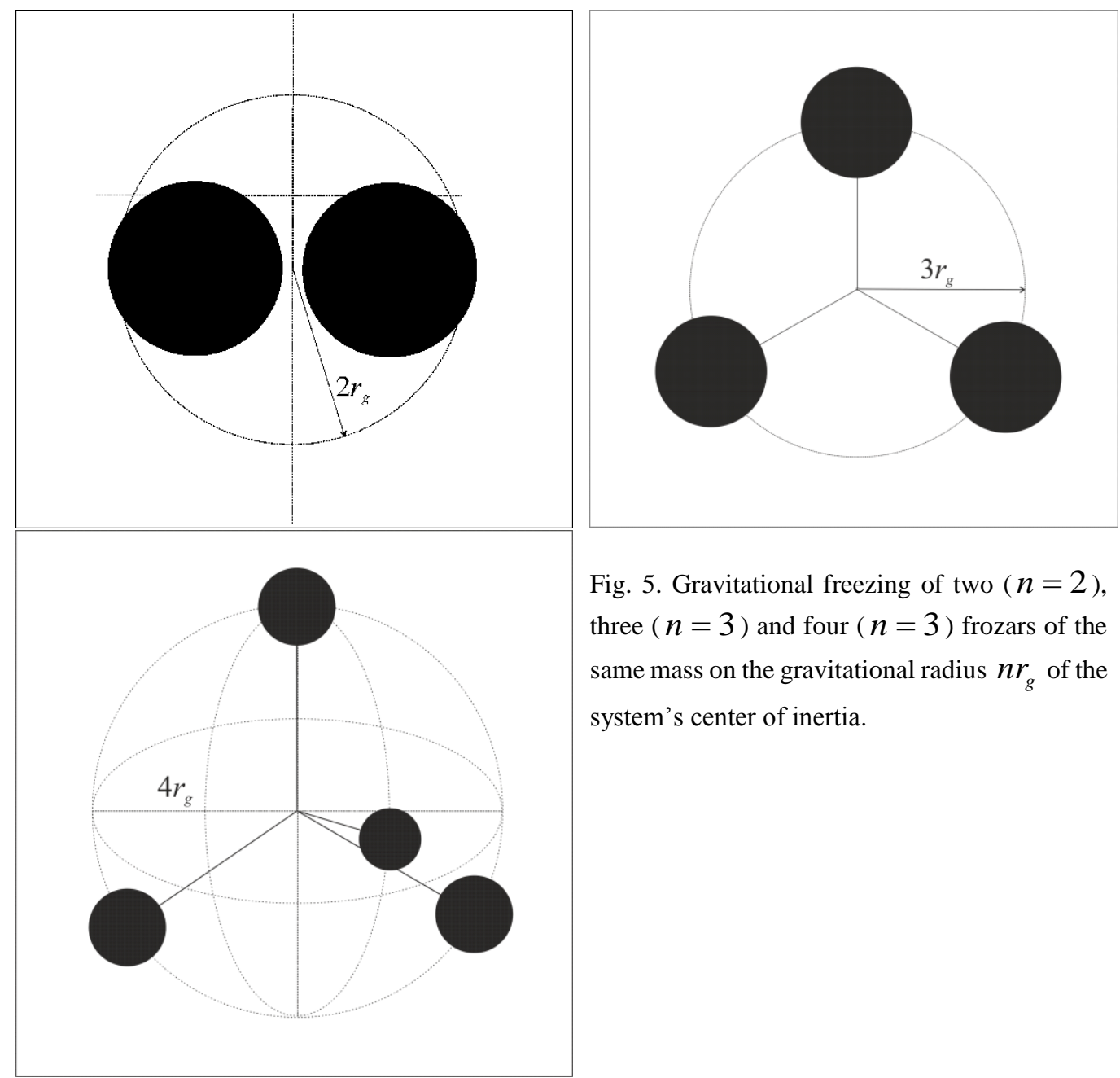

Fig. 5. Gravitational freezing of two $(n=2)$, three $(n=3)$ and four ( $n=3)$ frozars of the same mass on the gravitational radius $n r_{g}$ of the system's center of inertia.

\subsection{Supermassive frozars (superfrozars) as heterogeneous clusters}

The process of growth of frozars or their clusters during accretion with non-uniform freezing in some parts of the surface leads to the formation of a hybrid of frozars with an ordinary matter. This process can lead to the formation of very massive frozars - from the intermediate mass up to the supermassive frozars, or superfrozars. 
As a result, such gravitationally frozen supermassive clusters form a new state of matter, which seemingly initiated the formation of galaxies in earlier epochs or formed later due to the fall of stars and gas to the centers of galaxies.

In any of these cases, gravitationally frozen objects, significantly exceeding the mass of the Sun, have a complex and heterogeneous structure. This is due to the fact that during their formation massive falling objects, including whole stars, were destroyed only partially by tidal forces, and most of their material was flattened and frozen compactly on some parts of this cluster.

Usually, when an ordinary star is destroyed by the tidal forces of the stellar frozar, an accretion disk forms around the frozar. In the case of supermassive frozars, where tidal forces are small, ordinary stars and compact objects of stellar masses can be captured without loss of the most part of their matter and then fall almost radially, forming at the final stage the stellar mass mascons on the gravitational radius of the cluster.

\section{Observable effects of theory of frozars}

\subsection{Observable properties of frozars and explanation of known effects}

Frozars, unlike neutron stars, do not have a surface in the usual sense. The surface of the frozar freely falls at a local near-light speed, but be practically frozen in world time. This leads to the following effects for external observers.

Firstly, the surface of the frozar practically does not radiate, since locally emitted radiation will have such a large redshift that it will be almost elusive. Only the radially directed part of this super-weak radiation can leave the frozar field, while the entire nonradial part will be captured back.

Secondly, the frozar absorbs almost all the radiation falling on it, since the falling radiation can hardly "catch up" with the surface. Even an insignificant part that catches up and scatters on the particles of the surface will mainly be captured back. Nevertheless, in principle, the radial part of the radiation will be able to get out of the frozen region, although for a sufficiently long time.

Thirdly, particles and large objects falling on the frozar will be able to "catch up" with the surface only if their speed is greater than the speed on the surface for a given radial coordinate $r$. However, they freeze as well as the surface itself, since they fall into a region where, in terms of $t$, the time of the external world, practically "nothing happens".

These properties mean that frozar is not only a frozen star, but also an almost dark star. Nevertheless, frozar, even being almost dark, is not strictly black, but has a slight shade of gray, since radially directed radiation can in principle leave it.

For particles of finite mass, frozar is a very deep potential well, but it is not a hopeless "hole" from which there is no way out at all. Particles on the surface are outside $r_{g}$ and therefore those that acquire a radial speed close to the local speed of light will be able to move away from the frozar to a noticeable local distance, although almost all will fall back.

And, finally, unlike neutron stars, when a matter falls on a frozar, a flash of radiation will not be observed, since the falling occurs in the form of rapid freezing near the surface. Any outgoing radiation, as discussed above, will be so suppressed that for an external observer the falling to the frozar will be almost "silent".

These properties of frozars are in agreement with the observed properties of known compact objects, which are "candidates for frozars". 


\subsection{Effects of surface heterogeneity and internal structure}

The heterogeneity of the distribution of the matter density on the frozar surface, as a result of the inhomogeneous accretion created by the "mascon" of the mass $\delta m(\theta, \varphi)$, leads to small perturbations of the metric. In the field of a spherical frozar the perturbations $\delta g_{\mu \nu}(r, \theta, \varphi)$ will be added to the Schwarzschild metric over these regions of surface:

$$
\begin{aligned}
& g_{00}(r, \theta, \varphi)=g_{00}(r)+\delta g_{00}(r, \theta, \varphi), \\
& g_{11}(r, \theta, \varphi)=g_{11}(r)+\delta g_{11}(r, \theta, \varphi)
\end{aligned}
$$

These perturbations rapidly decrease with distance, but, nevertheless, lead to the observed effects. In some cases, for example, in the presence of orbiting sources of radiation, these inhomogeneities can be detected by means of gravimetry, as well as irregularities of redshifts and shadows.

In frozar gravimetry, the "normal" component of the gravitational potential $\phi(r)$ associated with the metric, like $g_{00}(r)=1+2 \phi(r)$ and the corresponding initially uniform surface of the frozar, should be separated from the angular perturbations $\delta \phi(r, \theta, \varphi)$ associated with inhomogeneous accretion of matter.

One of the observed consequences of such inhomogeneities in the metric is the appearance of small deviations in the shadow of the frozar. The gravitational deviation of light rays is distorted by perturbations of the metric, and for a ray passing at a distance $l$ from the center of the frozar, we have:

$$
\delta \varphi(\rho, \theta, \varphi) \simeq \frac{2 r_{g}}{l}+\frac{4 G \delta m(\theta, \varphi)}{l-r_{g}} \simeq \frac{2 r_{g}}{l}\left(1+\frac{\delta m(\theta, \varphi)}{m\left(1-r_{g} / l\right)}\right) .
$$

A stronger deviation of the rays from a more massive surface area increases the shadow border over this area, and the shadow border will contain irregularities.

Another class of observed effects is associated with the perturbation of redshifts and orbits of objects around the frozar. The standard expression for the frequency shift of photons becomes modified as:

$$
\omega(r, \theta, \varphi) \simeq \omega_{0}\left[1-\frac{r_{g}}{r}-\frac{2 G \delta m(\theta, \varphi)}{r-r_{g}}\right]^{1 / 2} \simeq \omega_{0}\left[1-\frac{r_{g}}{r}\left(1+\frac{\delta m(\theta, \varphi)}{m\left(1-r_{g} / r\right)}\right)\right]^{1 / 2} .
$$

Metric perturbations can also lead to an additional precession of the orbits, which can be observed from the radiation of an orbiting object.

\subsection{Consequences of the absence of an ergosphere in rotated frozars}

In Section 3.2 it was shown that in the static frame the surface of a rotating frozar freezes outside the outer gravitational radius of the Kerr metric Ошибка! Источник ссылки не найден., which was considered earlier as the outer boundary of the ergosphere. Since the metric inside such a frozar is given by a material solution and is not the Kerr metric, this means that the rotating frozar does not have an ergosphere. The former extrapolation of the vacuum solution, such as the Kerr solution, into the internal region of a collapsing star, had no logical basis and was only a mathematical exercise not having a physical meaning. 
The direct result of this fact is the absence of effects associated with the possible existence of the ergosphere, such as the extraction of rotational energy when particles enter the ergosphere by subsequent release.

\subsection{The effects of sticking of frozars without merging}

The result of sticking of frozars, discussed in Section 5.2, may be the largest heterogeneity in a cluster of frozars. These inhomogeneities also lead to observable effects that are stronger than the similar effects discussed above, such as mascons on the surface of the frozar. Gravimetry, as well as other methods, can also make it possible to study the internal structure of such frozar clusters.

In addition, the sticking of two frozars also leads to a shorter and weaker burst of emitted radiation at the final stage, including gravitational waves, than during merging. This issue requires further study with detailed numerical simulation of the process.

\subsection{Effects of a delay of falling the charged surface layer}

During the collapse of a charged dust star, charged particles concentrated on a thin surface layer, do not fall freely, but are decelerated by the electric field of the star. As a result, during the collapse, the star's surface layer lags behind the inner layers and falls at some distance from the neutral layers in the form of a thin charged shell or even freezes when the charge is sufficiently strong.

This layer is inhibited for some time at a finite distance above the star, which has become a frozar and then, falling onto the frozen region, the shell also freezes. If it stops without reaching the freezing region, then in astrophysics this situation does not last long time due to the gradual neutralization of the shell charge by the matter surrounding the star, after which the shell particles slowly settle onto the frozar.

The first class of observational effects of such a later fall of a charged shell is associated with the radiation of a charged shell and the interaction of radiation with this shell. The second class of effects is associated with the slow neutralization and the fall of this shell on the frozar with the disappearance of its effects.

For a rotating charged shell, magnetic field effects also appear, which slowly weaken as the shell is neutralized and settles.

\section{Conclusion}

The worldline of a particle on a thin dust shell and on the surface of a spherical dust star $r_{b}>r_{g}$ (and $\varphi, \theta=$ const. ) is described by $t\left(R_{b}, r_{b}\right)$ and $\tau\left(R_{b}, r_{b}\right)$, denoting the same event with the same particle. There is a one-to-one correspondence between both curves $t(R, r)$ and $\tau(R, r)$ representing the same event on the same worldline $r(R, t)=r(R, \tau)$, and therefore both curves are asymptotic. At $t \rightarrow \infty$ they approach the gravitational radius $r(R, t) \rightarrow r_{g}$ asymptotically and $r[R, \tau(t)] \rightarrow r_{g}$ (Fig. 1).

Diagrams of worldlines of star's particles (Fig. 2-3) show the internal structure of the dust star on the hypersurfaces of simultaneity $t=$ const. For large $t$ the worldlines of particles in all layers approach their asymptotes, parallel to the axis $t$, at internal effective gravitational radii. Layers that are equidistant at the beginning, during freezing, will also remain almost equidistant in the lower layers and densify only near the surface. 
This shows that the picture of a frozen star refers not only to the surface, asymptotically approaching the gravitational radius, remaining outside it, but also to the entire structure of the dust star. The picture of the structure and evolution of a star with asymptotically freezing layers on hypersurfaces simultaneity $t=$ const. will be reproduced in all frames of reference with physical coordinates, if matching with the external solution is done accurately.

Descriptions having physical meaning from the point of view of applying general relativity to astrophysics are those that at any finite moment of cosmological time in the vicinity of a star describe only those events in the inner layers that are simultaneous with the moment of world time $t$ on the surface. All other descriptions based on nonsimultaneous events in different parts of the star are either non-physical or can only play an auxiliary role for the subsequent transition to a set of simultaneous events in the entire volume of the star.

It is also important that in the OS solution the worldlines of star's particles cover every moment of the existence of these particles in the real world and, therefore, these solutions give us a complete picture of the evolution of the star. Irreversible freezing w.r.t. $t$ of all physical processes in the star due to the relativistic and gravitational dilation of its proper times is the physical phenomenon stopping the collapse. The presence of this fundamental physical phenomenon, the freezing of local processes, distinguishes relativistic collapse from the Newtonian, where there is no such stopping mechanism.

Thus, the collapse of the homogeneous dust star in general relativity in exact solutions by the OS method at any finite moment of time of the external world leads to a frozen star or frozar, an object having an almost uniform and practically frozen internal structure with time-like worldlines of particles of finite mass. The volume of the frozar, slightly larger than the gravitational radius, becomes a place where nothing happens on the time scales of the outside world.

The consideration of charged and rotating frozars made it possible to predict some of their new properties. For a charged dust star, a charged thin surface shell falls more slowly than internal neutral matter. There is no ergosphere in a rotating frozar, since its surface freezes at the outer boundary of the region where the ergosphere could form.

The observational consequences of the collapse with the formation at the final stage of the relativistic frozar turned out to be very diverse. One of them turned out to be the possible heterogeneity of the frozar field due to inhomogeneities on its surface generated by a matter that fell after the formation of the frozar and freeze on its surface. Another effect is the impossibility of merging the frozars and their sticking together due to the freezing of each of the frozars on the gravitational radius of the entire system.

A more general solution of the Einstein equations for the remaining two types of dust matter velocity, elliptic and hyperbolic $(k= \pm 1)$, at which the local homogeneity is preserved, is presented in the second paper [7].

\section{References}

1. Oppenheimer J., Snyder H. (1939) Phys. Rev., 56, 455.

2. Tolman R. (1934) Proc. N. Acad. Sci . USA, 20, 169.

3. Zakir Z. (2017) TPAC, 12(1) 1; 12(1) 17.

4. Zakir Z. (2018) Astroph. \& Space Sci. 363: 30.

5. Klein O. (1961). Ein. Prob. allgem Rel. in W. Heisenb. Phys. unz. Z., p. 58. 
6. Weinberg S. (1972). Gravitation and Cosmology. Wiley.

7. Zakir Z. (2020) Quant. and Grav. Phys., 1:007-7131.

8. Zakir Z. TPAC, (2006) 1(3) 45; (2007) 2(1) 1 (arxiv:0705.2585).

9. Zakir Z. TPAC, (2012) 7(1) 1; 7(1) 15; 7(1) 15; (2015) 10(1) 43.

10. Zakir Z. (2012) TPAC, 7(2) 23.

11. Zakir Z. (2020) Theory of Frozars. Relativistic Collapse of Stars. CTPA, T.

12. Landau L. D., Lifshitz E. M. (1987) The Classical Theory of Fields. B. H.

13. Kerr R. (1963) Phys. Rev. Lett., 11, 237.

14. Marshall T. (2012) Astroph. \& Space Sci., 342:329. 